\title{
Weak approximation of the Brownian sheet from a Poisson process in the plane
}

\author{
XAVIER BARDINA* and MARIA JOLIS** \\ Departament de Matemàtiques, Edifici C, Universitat Autònoma de Barcelona, 08193 Bellaterra, \\ Barcelona, Spain.E-mail: *bardina@mat.uab.es, ${ }^{* *}$ mjolis@mat.uab.es
}

We show an approximation in law of the Brownian sheet by processes constructed from the Poisson process in the plane. This result was inspired by a similar result of Stroock in the one-parameter case.

Keywords: two-parameter Poisson process; two-parameter Wiener process; weak convergence

\section{Introduction and main result}

The purpose of this paper is to prove a weak convergence to the Brownian sheet result for processes constructed from a two-parameter Poisson process. We seek an analogous result, in the two-parameter case, of the following theorem proved by Stroock (1982).

Theorem. Consider a standard Poisson process, $\{N(t), t \geqslant 0\}$, and define, for any $\varepsilon>0$, the continuous processes

$$
y_{\varepsilon}=\left\{y_{\varepsilon}(t):=\varepsilon \int_{0}^{t / \varepsilon^{2}}(-1)^{N(s)} \mathrm{d} s, t \in[0, T]\right\} .
$$

If $\left(P^{\varepsilon}\right)$ are the laws of the $y_{\varepsilon}$ in the Banach space $\mathscr{C}([0, T])$ of continuous functions on $[0, T]$, then $\left(P^{\varepsilon}\right)$ converges weakly, as $\varepsilon$ tends to zero, towards the Wiener measure.

A motivation for proving results of this type is that they provide examples of processes of finite variation that can be approximated in law by the Wiener process. These processes have very different properties from the classical examples constructed from sums of independent random variables and from stationary processes, which also converge in law to the Wiener process.

Another point of interest is that they give a nice relationship between the two more important processes.

Our result is the following: 
Theorem 1.1. Define

$$
\left\{x_{\varepsilon}(s, t):=\varepsilon \int_{0}^{t / \varepsilon} \int_{0}^{s / \varepsilon} \sqrt{x y}(-1)^{N(x, y)} \mathrm{d} x \mathrm{~d} y ;(s, t) \in[0, S] \times[0, T]\right\},
$$

where $\{N(x, y) ; x, y \geqslant 0\}$ is a Poisson process in the plane.

Consider $P_{\varepsilon}$ the image law of $x_{\varepsilon}$ in the Banach space $\mathscr{C}([0, S] \times[0, T])$ of continuous functions on $[0, S] \times[0, T]$. Then, $\left(P_{\varepsilon}\right)$ converges weakly, as $\varepsilon$ tends to zero, towards the law on $\mathscr{C}([0, S] \times[0, T])$ of a Brownian sheet.

The integrand $(-1)^{N(x, y)}$ changes the sign very quickly if there are a lot of points around it. So, when $\varepsilon$ tends to zero, $(-1)^{N(x / \varepsilon, y / \varepsilon)}$ tends to something which has independent values at each point and, properly normalized, is approximately white noise. by

One might expect that the result in the two-parameter case was that the processes defined

$$
Y_{\varepsilon}(s, t):=\varepsilon \int_{0}^{t / \varepsilon} \int_{0}^{s / \varepsilon}(-1)^{N(x, y)} \mathrm{d} x \mathrm{~d} y
$$

converge weakly to the Brownian sheet. But it can be proved that the $Y_{\varepsilon}(s, t)$ converge to zero, as $\varepsilon$ tends to zero, in $L^{2}(\Omega)$, for all $(s, t) \in[0, S] \times[0, T]$.

An intuitive reason for this apparent pathology is that the speed of convergence is not the same for all points $(x, y)$. It is slower near the origin than further away, and the square root factor expresses that. Another reason is the following.

We can write the Stroock processes as

$$
y_{\varepsilon}(t)=\int_{0}^{t} \frac{1}{\varepsilon}(-1)^{N\left(s / \varepsilon^{2}\right)} \mathrm{d} s,
$$

the process defined in Theorem 1.1 as

$$
x_{\varepsilon}(s, t)=\int_{0}^{t} \int_{0}^{s} \frac{1}{\varepsilon^{2}} \sqrt{x y}(-1)^{N(x / \varepsilon, y / \varepsilon)} \mathrm{d} x \mathrm{~d} y
$$

and

$$
Y_{\varepsilon}(s, t)=\int_{0}^{t} \int_{0}^{s} \frac{1}{\varepsilon}(-1)^{N(x / \varepsilon, y / \varepsilon)} \mathrm{d} x \mathrm{~d} y .
$$

If we consider the covariance function of the integrand process in the expression of $y_{\varepsilon}$,

$$
\begin{aligned}
K_{\varepsilon}\left(t, t^{\prime}\right) & =\mathrm{E}\left[\frac{1}{\varepsilon^{2}}(-1)^{N\left(t / \varepsilon^{2}\right)}(-1)^{N\left(t^{\prime} / \varepsilon^{2}\right)}\right]-\frac{1}{\varepsilon^{2}} \mathrm{E}\left[(-1)^{N\left(t / \varepsilon^{2}\right)}\right] \mathrm{E}\left[(-1)^{N\left(t^{\prime} / \varepsilon^{2}\right)}\right] \\
& =\frac{1}{\varepsilon^{2}} \exp \left[-\frac{2\left|t-t^{\prime}\right|}{\varepsilon^{2}}\right]-\frac{1}{\varepsilon^{2}} \exp \left[-\frac{2\left(t+t^{\prime}\right)}{\varepsilon^{2}}\right],
\end{aligned}
$$

it is clear that, for any $t>0$, as a function of $t^{\prime}$, this covariance converges weakly, as $\varepsilon$ tends 
to zero, to $\delta_{t}$, the unit mass measure at the point $t$ (which is the 'covariance function' of the white noise).

On the other hand, if we compute the covariance for the integrand processes in the expression of $Y_{\varepsilon}$ and $x_{\varepsilon}$, we can see that $K_{\varepsilon}^{Y}\left((s, t),\left(s^{\prime}, t^{\prime}\right)\right)$ as a function of $\left(s^{\prime}, t^{\prime}\right)$ converges weakly to zero, while $K_{\varepsilon}^{x}\left((s, t),\left(s^{\prime}, t^{\prime}\right)\right)$ tends weakly to $\delta_{(s, t)}$. These two last facts can be proved by similar arguments to those used in the proof of Lemma 4.4 below.

In order to simplify the notation we denote by $N_{\mu}(x, y)$ the random variable $N(x \sqrt{\mu}, y \sqrt{\mu})$. Then $\left\{N_{\mu}(x, y) ;(x, y) \in \mathbb{R}_{+}^{2}\right\}$ is a Poisson process with intensity $\mu$. Note that

$$
x_{\varepsilon}(s, t)=\frac{1}{\varepsilon^{2}} \int_{0}^{t} \int_{0}^{s} \sqrt{x y}(-1)^{N_{1 / \varepsilon^{2}}(x, y)} \mathrm{d} x \mathrm{~d} y .
$$

Setting $n=1 / \varepsilon^{2}$, we are looking for the weak limit as $n \rightarrow \infty$ of

$$
x_{n}(s, t):=n \int_{0}^{t} \int_{0}^{s} \sqrt{x y}(-1)^{N_{n}(x, y)} \mathrm{d} x \mathrm{~d} y,
$$

and we denote by $P_{n}$ the image law of $x_{n}$ in the space $\mathscr{C}([0, S] \times[0, T])$.

The paper is organized as follows. Section 2 is devoted to some preliminaries on twoparameter processes. The proof of tightness of the family of laws $\left(P_{n}\right)$ is given in Section 3 . Finally, in Section 4, we identify all the possible weak limits of subsequences of $\left(P_{n}\right)$ as the Wiener measure.

A lot of the estimates in the paper contain constants (not depending on $n$ ). We use the same letter, $K$, for these constants, although their actual value can vary from one expression to the next.

\section{Preliminaries}

We will use the notation and definitions introduced in the basic work of Cairoli and Walsh (1975) on stochastic calculus in the plane. We recall some of them here.

Let $(\Omega, \mathscr{F}, P)$ be a complete probability space and let $\left\{\mathscr{F}_{s, t} ;(s, t) \in[0, S] \times[0, T]\right\}$ be a family of sub- $\sigma$-fields of $\mathscr{F}$ such that:

(i) $\mathscr{F}_{s, t} \subseteq \mathscr{F}_{s^{\prime}, t^{\prime}}$ for any $s \leqslant s^{\prime}, t \leqslant t^{\prime}$;

(ii) $\mathscr{F} 0,0$ contains all null sets of $\mathscr{F}$;

(iii) for each $z \in[0, S] \times[0, T], \mathscr{F}_{z}=\cap_{z<z^{\prime}} \mathscr{F}_{z^{\prime}}$, where $z=(s, t)<z^{\prime}=\left(s^{\prime}, t^{\prime}\right)$ denotes the partial order on $[0, S] \times[0, T]$, meaning that $s<s^{\prime}$ and $t<t^{\prime}$.

Given $(s, t)<\left(s^{\prime}, t^{\prime}\right)$, we denote by $\Delta_{s, t} X_{s^{\prime}, t^{\prime}}$ the increment of the process $X$ over the rectangle $\left((s, t),\left(s^{\prime}, t^{\prime}\right)\right]$, that is,

$$
\Delta_{s, t} X_{s^{\prime}, t^{\prime}}=X_{s^{\prime}, t^{\prime}}-X_{s, t^{\prime}}-X_{s^{\prime}, t}+X_{s, t} .
$$

It is said that an $\mathscr{F}_{z}$-adapted process $X=\left\{X_{z} ; z \in[0, S] \times[0, T]\right\}$ is a martingale if $\mathrm{E}\left(\left|X_{z}\right|\right)<\infty$ for all $z \in[0, S] \times[0, T]$ and 


$$
\mathrm{E}\left(X_{s^{\prime}, t^{\prime}}-X_{s, t} \mid \mathscr{F}_{s, t}\right)=0, \quad \text { for any }(s, t)<\left(s^{\prime}, t^{\prime}\right)
$$

It is said that an $\mathscr{F}_{z}$-adapted process $X=\left\{X_{z} ; z \in[0, S] \times[0, T]\right\}$ is a strong martingale if $\mathrm{E}\left(\left|X_{z}\right|\right)<\infty$ for all $z \in[0, S] \times[0, T], X_{s, 0}=X_{0, s}=0$ for all $s \geqslant 0$ and

$$
\mathrm{E}\left(\Delta_{s, t} X_{s^{\prime}, t^{\prime}} \mid \mathscr{F}_{S, t} \vee \mathscr{F}_{s, T}\right)=0, \quad \text { for any }(s, t)<\left(s^{\prime}, t^{\prime}\right) .
$$

Definition 2.1. An $\mathscr{F}_{s, t}$-Brownian sheet is a continuous, adapted process $W=\left\{W_{s, t}\right.$; $(s, t) \in[0, S] \times[0, T]\} \quad$ such that $W_{s, 0}=W_{0, t}=0$ almost surely (a.s.), for all $(s, t) \leqslant\left(s^{\prime}, t^{\prime}\right)$, the increment $\Delta_{s, t} W_{s^{\prime}, t^{\prime}}$ is independent of $\mathscr{F}_{s, t} \vee \mathscr{F}_{s, T}$ and is normally distributed with zero mean and variance $\left(s^{\prime}-s\right)\left(t^{\prime}-t\right)$.

If we do not specify the filtration, $\left(\mathscr{F}_{s, t}\right)$ will be the filtration generated by the process itself, completed with the null sets of $\mathscr{F}^{W}=\sigma\left\{W_{s, t},(s, t) \in[0, S] \times[0, T]\right\}$.

Definition 2.2. Let $\left\{\mathscr{F}_{s, t}\right\}$ be a family of sub- $\sigma$-fields of $\mathscr{F}$ satisfying the previous conditions for all $(s, t) \in \mathbb{R}_{+}^{2}$. An $\mathscr{F}_{s, t}$-Poisson process is an adapted, cadlag process $N=\left\{N_{s, t}\right.$; $\left.(s, t) \in \mathbb{R}_{+}^{2}\right\}$, such that, $N_{s, 0}=N_{0, t}=0$ a.s., for all $(s, t) \leqslant\left(s^{\prime}, t^{\prime}\right)$ the increment $\Delta_{s, t} N_{s^{\prime}, t^{\prime}}$ is independent of $\mathscr{F}_{\infty, t} \vee \mathscr{F}_{s, \infty}$ and has a Poisson law of parameter $\left(s^{\prime}-s\right)\left(t^{\prime}-t\right)$. Here, we are denoting $\mathscr{F}_{\infty, t}:=\vee_{s>0} \mathscr{F}_{s, t}$ and $\mathscr{F}_{s, \infty}:=\vee_{t>0} \mathscr{F}_{s, t}$.

If we do not specify the filtration, $\left(\mathscr{F}_{s, t}\right)$ will be the filtration generated by the process itself, completed with the nulls sets of $\mathscr{F}^{N}=\sigma\left\{N_{s, t},(s, t) \in \mathbb{R}_{+}^{2}\right\}$.

\section{Proof of tightness}

To prove Theorem 1.1, we have to check that the family $P_{n}$ is tight and that any weakly convergent subsequence converges to the law of a Brownian sheet. In this section we prove that $P_{n}$ is tight. Using the criterion given by Bickel and Wichura (1971), and that our processes $x_{n}$ are null on the axes, it suffices to prove the following lemma.

Lemma 3.1. Let $\left\{x_{n}\right\}$ be the family of processes defined by (1). There exists a constant $K$ such that, for any $(s, t)<\left(s^{\prime}, t^{\prime}\right)$,

$$
\sup _{n} \mathrm{E}\left[\left(\Delta_{s, t} x_{n}\left(s^{\prime}, t^{\prime}\right)\right)^{4}\right] \leqslant K\left(s^{\prime}-s\right)^{2}\left(t^{\prime}-t\right)^{2} .
$$

In order to prove Lemma 3.1 it will be useful to have the following result which we will also utilize in Section 4.

Lemma 3.2. Let $\left\{x_{n}\right\}$ be the family of processes defined by (1). Then if $(s, t)<\left(s^{\prime}, t^{\prime}\right)$,

$$
\mathrm{E}\left[\left(\Delta_{s, t} x_{n}\left(s^{\prime}, t^{\prime}\right)\right)^{2}\right] \leqslant 4\left(s^{\prime}-s\right)\left(t^{\prime}-t\right)
$$




\section{Proof.}

$$
\begin{aligned}
\mathrm{E}\left[\left(\Delta_{s, t} x_{n}\left(s^{\prime}, t^{\prime}\right)\right)^{2}\right] & =n^{2} \mathrm{E}\left[\left(\int_{t}^{t^{\prime}} \int_{s}^{s^{\prime}} \sqrt{x y}(-1)^{N_{n}(x, y)} \mathrm{d} x \mathrm{~d} y\right)^{2}\right] \\
& =n^{2} \mathrm{E}\left[\prod_{i=1}^{2}\left(\int_{t}^{t^{\prime}} \int_{s}^{s^{\prime}} \sqrt{x_{i} y_{i}}(-1)^{N_{n}\left(x_{i}, y_{i}\right)} \mathrm{d} x_{i} \mathrm{~d} y_{i}\right)\right] \\
& =n^{2} \int_{\left[s, s^{\prime}\right]^{2} \times\left[t, t^{\prime}\right]^{2}} \sqrt{x_{1} x_{2} y_{1} y_{2}} \mathrm{E}\left[(-1)^{N_{n}\left(x_{1}, y_{1}\right)+N_{n}\left(x_{2}, y_{2}\right)}\right] \mathrm{d} x_{1} \mathrm{~d} x_{2} \mathrm{~d} y_{1} \mathrm{~d} y_{2} .
\end{aligned}
$$

Observe that $(-1)^{\sum_{i=1}^{2} N_{n}\left(x_{i}, y_{i}\right)}=(-1)^{\sum_{i=1}^{2} \Delta_{0,0} N_{n}\left(x_{i}, y_{i}\right)}$, and this last sum is equal to the sum of the increments of the Poisson process over some disjoint rectangles. Each one of these last increments appears once or twice. Obviously the rectangles which contribute to the value of $(-1)^{\sum_{i=1}^{2} \Delta_{0,0} N_{n}\left(x_{i}, y_{i}\right)}$ are those that appear only once.

If we suppose that $x_{1} \leqslant x_{2}$, there are two possible orders in the plane for the points $\left(x_{1}, y_{1}\right),\left(x_{2}, y_{2}\right)$. (See Figure 1, where the black zones correspond to the rectangles that appear only once in the sum $\sum_{i=1}^{2} \Delta_{0,0} N_{n}\left(x_{i}, y_{i}\right)$.)

Now, using the fact that the Poisson process has independent increments, and that if $Z \sim \operatorname{Poiss}(\lambda)$ then $\left.\mathrm{E}\left[(-1)^{Z}\right]\right)=\exp (-2 \lambda)$, we obtain that

$$
\mathrm{E}\left[\left(\Delta_{s, t} x_{n}\left(s^{\prime}, t^{\prime}\right)\right)^{2}\right]=2\left(I_{1}+I_{2}\right)
$$

where
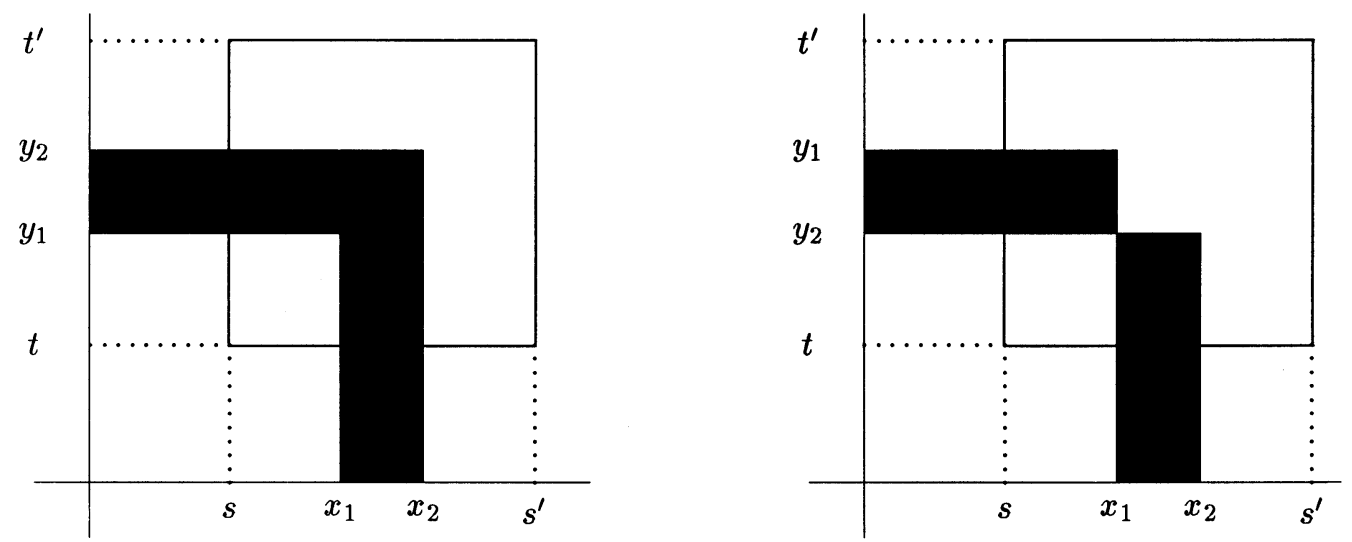

Figure 1. The two possible orders of two points in the plane. 


$$
\begin{aligned}
I_{1}= & n^{2} \int_{\left[s, s^{\prime}\right]^{2} \times\left[t, t^{\prime}\right]^{2}} \sqrt{x_{1} x_{2} y_{1} y_{2}} \exp \left[-2 n\left(x_{2} y_{2}-x_{1} y_{1}\right)\right] I_{\left\{x_{1} \leqslant x_{2}\right\}} I_{\left\{y_{1} \leqslant y_{2}\right\}} \mathrm{d} x_{1} \mathrm{~d} x_{2} \mathrm{~d} y_{1} \mathrm{~d} y_{2}, \\
I_{2}= & n^{2} \int_{\left[s, s^{\prime}\right]^{2} \times\left[t, t^{\prime}\right]^{2}} \sqrt{x_{1} x_{2} y_{1} y_{2}} \exp \left[-2 n\left(x_{2}-x_{1}\right) y_{2}-2 n\left(y_{1}-y_{2}\right) x_{1}\right] \\
& \times I_{\left\{x_{1} \leqslant x_{2}\right\}} I_{\left\{y_{2} \leqslant y_{1}\right\}} \mathrm{d} x_{1} \mathrm{~d} x_{2} \mathrm{~d} y_{1} \mathrm{~d} y_{2} .
\end{aligned}
$$

By doing a change of variables in order to obtain $y_{1} \leqslant y_{2}$, it is easy to see that $I_{1} \leqslant I_{2}$. Then,

$$
\begin{aligned}
\mathrm{E}\left[\left(\Delta_{s, t} x_{n}\left(s^{\prime}, t^{\prime}\right)\right)^{2}\right] \leqslant & 4 n^{2} \int_{\left[s, s^{\prime}\right]^{2} \times\left[t, t^{\prime}\right]^{2}} \sqrt{x_{1} x_{2} y_{1} y_{2}} \exp \left[-2 n\left(x_{2}-x_{1}\right) y_{1}-2 n\left(y_{2}-y_{1}\right) x_{1}\right] \\
& \times I_{\left\{x_{1} \leqslant x_{2}\right\}} I_{\left\{y_{1} \leqslant y_{2}\right\}} \mathrm{d} x_{1} \mathrm{~d} x_{2} \mathrm{~d} y_{1} \mathrm{~d} y_{2} .
\end{aligned}
$$

By using the fact that $x_{2} \leqslant s^{\prime}, y_{2} \leqslant t^{\prime}$ and then integrating with respect to these two variables, we obtain that the last expression is less than or equal to

$$
\begin{aligned}
\sqrt{s^{\prime}} \sqrt{t^{\prime}} \int_{s}^{s^{\prime}} \frac{1}{\sqrt{x_{1}}} \mathrm{~d} x_{1} \int_{t}^{t^{\prime}} \frac{1}{\sqrt{y_{1}}} \mathrm{~d} y_{1} & =4 \sqrt{s^{\prime}}\left(\sqrt{s^{\prime}}-\sqrt{s}\right) \sqrt{t^{\prime}}\left(\sqrt{t^{\prime}}-\sqrt{t}\right) \\
& \leqslant 4\left(s^{\prime}-s\right)\left(t^{\prime}-t\right) .
\end{aligned}
$$

We are now ready to prove Lemma 3.1.

Proof of Lemma 3.1. By arguments of additivity it is enough to prove the lemma for the case where $s$ and $t$ are strictly positive, and $t^{\prime}-t<t$ and $s^{\prime}-s<s$.

We have that

$$
\begin{aligned}
\mathrm{E}\left[\left(\Delta_{s, t} x_{n}\left(s^{\prime}, t^{\prime}\right)\right)^{4}\right] & =n^{4} \mathrm{E}\left[\left(\int_{t}^{t^{\prime}} \int_{s}^{s^{\prime}} \sqrt{x y}(-1)^{N_{n}(x, y)} \mathrm{d} x \mathrm{~d} y\right)^{4}\right] \\
& =n^{4} \mathrm{E}\left[\prod_{i=1}^{4}\left(\int_{t}^{t^{\prime}} \int_{s}^{s^{\prime}} \sqrt{x_{i} y_{i}}(-1)^{N_{n}\left(x_{i} y_{i}\right)} \mathrm{d} x_{i} \mathrm{~d} y_{i}\right)\right] .
\end{aligned}
$$

Observe that $(-1)^{\sum_{i=1}^{4} N_{n}\left(x_{i}, y_{i}\right)}=(-1)^{\sum_{i=1}^{4} \Delta_{0,0} N_{n}\left(x_{i}, y_{i}\right)}$, and that

$$
\sum_{i=1}^{4} \Delta_{0,0} N_{n}\left(x_{i}, y_{i}\right)=\sum_{i=1}^{4} \Delta_{s, t} N_{n}\left(x_{i}, y_{i}\right)+\sum_{i=1}^{4} \Delta_{s, 0} N_{n}\left(x_{i}, t\right)+\sum_{i=1}^{4} \Delta_{0, t} N_{n}\left(s, y_{i}\right)+4 \Delta_{0,0} N_{n}(s, t) .
$$

So,

$$
(-1)^{\sum_{i=1}^{4} \Delta_{0,0} N_{n}\left(x_{i}, y_{i}\right)}=(-1)^{\sum_{i=1}^{4} \Delta_{s, t} N_{n}\left(x_{i}, y_{i}\right)}(-1)^{\sum_{i=1}^{4} \Delta_{s, 0} N_{n}\left(x_{i}, t\right)}(-1)^{\sum_{i=1}^{4} \Delta_{0, t} N_{n}\left(s, y_{i}\right)},
$$


and these three factors are independent. If we suppose $x_{1} \leqslant x_{2} \leqslant x_{3} \leqslant x_{4}$ and $y_{1} \leqslant y_{2} \leqslant$ $y_{3} \leqslant y_{4}$, we have that

$$
\begin{aligned}
\mathrm{E}\left[(-1)^{\sum_{i=1}^{4} \Delta_{s, 0} N_{n}\left(x_{i}, t\right)}\right] \mathrm{E}\left[(-1)^{\sum_{i=1}^{4} \Delta_{0, t} N_{n}\left(s, y_{i}\right)}\right] & \\
& =\exp \left[-2 n t\left[\left(x_{4}-x_{3}\right)+\left(x_{2}-x_{1}\right)\right] \exp \left[-2 n s\left[\left(y_{4}-y_{3}\right)+\left(y_{2}-y_{1}\right)\right] ;\right.\right.
\end{aligned}
$$

by using the fact that $2 t>t^{\prime}$ and $2 s>s^{\prime}$, the last expression is less than or equal to

$$
\begin{aligned}
\exp \left[-n t^{\prime}\left[\left(x_{4}-x_{3}\right)+\right.\right. & \left.\left(x_{2}-x_{1}\right)\right] \exp \left[-n s^{\prime}\left[\left(y_{4}-y_{3}\right)+\left(y_{2}-y_{1}\right)\right]\right. \\
& \leqslant \exp \left[-n\left[\left(x_{4}-x_{3}\right) y_{3}+\left(x_{2}-x_{1}\right) y_{1}\right] \exp \left[-n\left[\left(y_{4}-y_{3}\right) x_{3}+\left(y_{2}-y_{1}\right) x_{1}\right]\right.\right.
\end{aligned}
$$

Finally, we can bound $\mathrm{E}\left[(-1)^{\sum_{i=1}^{4} \Delta_{s, t} N_{n}\left(x_{i}, y_{i}\right)}\right]$ by 1 . So,

$$
\begin{aligned}
\mathrm{E}\left[\left(\Delta_{s, t} x_{n}\left(s^{\prime}, t^{\prime}\right)\right)^{4}\right] \leqslant & K n^{4} \int_{\left[s, s^{\prime}\right]^{4} \times\left[t, t^{\prime}\right]^{4}} \prod_{i=1}^{4} \sqrt{x_{i} y_{i}} \exp \left[-n\left(x_{4}-x_{3}\right) y_{3}+n\left(x_{2}-x_{1}\right) y_{1}\right] \\
& \times I_{\left\{x_{1} \leqslant x_{2} \leqslant x_{3} \leqslant x_{4}\right\}} \exp \left[-n\left(y_{4}-y_{3}\right) x_{3}+n\left(y_{2}-y_{1}\right) x_{1}\right] I_{\left\{y_{1} \leqslant y_{2} \leqslant y_{3} \leqslant y_{4}\right\}} \mathrm{d} x_{1} \ldots \mathrm{d} y_{4} \\
\leqslant & K\left(n^{2} \int_{\left[s, s^{\prime}\right]^{2} \times\left[t, t^{\prime}\right]^{2}} \sqrt{x_{1} x_{2} y_{1} y_{2}} \exp \left[-n\left(x_{2}-x_{1}\right) y_{1}-n\left(y_{2}-y_{1}\right) x_{1}\right]\right. \\
& \left.\times I_{\left\{x_{1} \leqslant x_{2}\right\}} I_{\left\{y_{1} \leqslant y_{2}\right\}} \mathrm{d} x_{1} \mathrm{~d} x_{2} \mathrm{~d} y_{1} \mathrm{~d} y_{2}\right)^{2} \\
\leqslant & K\left(n^{2} \int_{\left[\frac{s}{2}, s_{2}^{\prime}\right]^{2} \times[t, t]^{2}} \sqrt{x_{1} x_{2} y_{1} y_{2}} \exp \left[-2 n\left(x_{2}-x_{1}\right) y_{1}-2 n\left(y_{2}-y_{1}\right) x_{1}\right]\right. \\
& \left.\times I_{\left\{x_{1} \leqslant x_{2}\right\}} I_{\left\{y_{1} \leqslant y_{2}\right\}} \mathrm{d} x_{1} \mathrm{~d} x_{2} \mathrm{~d} y_{1} \mathrm{~d} y_{2}\right)^{2} .
\end{aligned}
$$

Using the computations of Lemma 3.2, the last expression is bounded by $K\left(t^{\prime}-t\right)^{2}\left(s^{\prime}-s\right)^{2}$.

\section{Identification of the limit law}

We have proved that the family $P_{n}$ is tight. Now, we must show that the law of all possible weak limits is the law of a Brownian sheet.

Let $\left\{P_{n_{i}}\right\}_{i}$ be a subsequence of $\left\{P_{n}\right\}_{n}$ (which we will also denote by $\left\{P_{n}\right\}$ ) weakly convergent to some probability $P$. We want to show that $P$ is the Wiener measure, that is, the canonical process $\left\{X_{s, t}(x)=: x(s, t)\right\}$ is a Brownian sheet under the probability $P$.

There exist various possible characterizations of a Brownian sheet; see, for example, Tudor (1980) or Florit and Nualart (1996). In particular, in Theorem 2.2 of Florit and Nualart (1996) necessary and sufficient conditions for a process to be a Brownian motion, with respect to an arbitrary filtration, are proved. If we just consider the case in with the underlying filtration is the natural one, we realize that we can weaken the hypotheses of 
Theorem 2.2 of Florit and Nualart (1996). Then, we obtain the following characterization of the two-parameter Wiener process.

Theorem 4.1. Let $X=\left\{X_{s, t} ;(s, t) \in[0, S] \times[0, T]\right\}$ be a continuous process such that $X_{s, 0}=X_{0, t}=0$. And let $\left(\mathscr{F}_{s, t}\right)$ be the natural filtration of $X$.

Then, the following statements are equivalent:

(i) $X$ is a Brownian sheet.

(ii) For any $0<s \leqslant s^{\prime}, \quad 0<t \leqslant t^{\prime}, \quad \mathrm{E}\left(\Delta_{s, t} X_{s^{\prime}, t^{\prime}} \mid \mathscr{F}_{S, t} \vee \mathscr{F}_{s, T}\right)=0 \quad$ and $\mathrm{E}\left[\left(\Delta_{s, t} X_{s^{\prime}, t^{\prime}}\right)^{2} \mid\right.$ $\left.\mathscr{F}_{S, t} \vee F_{S, T}\right]=\left(s^{\prime}-s\right)\left(t^{\prime}-t\right)$.

The difference between this theorem and Theorem 2.2 of Florit and Nualart (1996) is that in order to obtain a Brownian sheet (with respect to its natural filtration) we need only to check the properties of strong martingale and 'quadratic variation' for increments over rectangles without intersection with axes.

So, in order to prove that the limit law is the Wiener measure, it suffices to prove the following two propositions.

Proposition 4.2. Suppose that $\left\{P_{n}\right\}$ are the laws in $\mathscr{C}([0, S] \times[0, T])$ of the processes $x_{n}$ defined by (1), and assume that $\left\{P_{n_{i}}\right\}$ is a subsequence weakly convergent to $P$. Let $X$ be the canonical process and let $\left\{\mathscr{F}_{s, t}\right\}$ be its natural filtration. Then for any $0<s \leqslant s^{\prime}, 0<t \leqslant t^{\prime}$, $\mathrm{E}_{P}\left(\Delta_{s, t} X_{s^{\prime}, t^{\prime}} \mid \mathscr{F}_{S, t} \vee \mathscr{F}_{s, T}\right)=0$.

Proposition 4.3. Under the hypotheses of the above proposition, we have that

$$
\mathrm{E}_{P}\left[\left(\Delta_{s, t} X_{s^{\prime}, t^{\prime}}\right)^{2} \mid \mathscr{F}_{S, t} \vee \mathscr{F}_{s, T}\right]=\left(s^{\prime}-s\right)\left(t^{\prime}-t\right), \quad \text { for any } 0<s \leqslant s^{\prime}, 0<t \leqslant t^{\prime} .
$$

Proof of Proposition 4.2. We only need to prove that for any $\left(s_{1}, t_{1}\right), \ldots,\left(s_{m}, t_{m}\right)$ and $\delta>0$, with $s_{i} \leqslant S, t_{i} \leqslant t-\delta$ or $s_{i} \leqslant s-\delta, t_{i} \leqslant T$, for $i=1, \ldots, m$, and for any bounded continuous $\varphi: \mathbb{R}^{m} \rightarrow \mathbb{R}$

$$
\mathrm{E}_{P}\left[\varphi\left(X_{s_{1}, t_{1}}, \ldots, X_{s_{m}, t_{m}}\right)\left(\Delta_{s, t} X_{s^{\prime}, t^{\prime}}\right)\right]=0 .
$$

Since $P_{n} \stackrel{w}{\Rightarrow} P$, and taking into account Lemma 3.1 , we have that

$\lim _{n \rightarrow \infty} \mathrm{E}_{P_{n}}\left[\varphi\left(x\left(s_{1}, t_{1}\right), \ldots, x\left(s_{m}, t_{m}\right)\right)\left(\Delta_{s, t} x\left(s^{\prime}, t^{\prime}\right)\right)\right]=\mathrm{E}_{P}\left[\varphi\left(x\left(s_{1}, t_{1}\right), \ldots, x\left(s_{m}, t_{m}\right)\right)\left(\Delta_{s, t} x\left(s^{\prime}, t^{\prime}\right)\right)\right]$.

Thus, it suffices to prove that

$$
\lim _{n \rightarrow \infty} \mathrm{E}_{P_{n}}\left[\varphi\left(x\left(s_{1}, t_{1}\right), \ldots, x\left(s_{m}, t_{m}\right)\right)\left(\Delta_{s, t} x\left(s^{\prime}, t^{\prime}\right)\right)\right]=0 .
$$

We have that 


$$
\begin{aligned}
\mid \mathrm{E}_{P_{n}} & {\left[\varphi\left(x\left(s_{1}, t_{1}\right), \ldots, x\left(s_{m}, t_{m}\right)\right)\left(\Delta_{s, t} x\left(s^{\prime}, t^{\prime}\right)\right)\right] \mid } \\
& =\left|\mathrm{E}\left[\varphi\left(x_{n}\left(s_{1}, t_{1}\right), \ldots, x_{n}\left(s_{m}, t_{m}\right)\right)\left(\Delta_{s, t} x_{n}\left(s^{\prime}, t^{\prime}\right)\right)\right]\right| \\
& =\left|\mathrm{E}\left[\varphi\left(x_{n}\left(s_{1}, t_{1}\right), \ldots, x_{n}\left(s_{m}, t_{m}\right)\right) \mathrm{E}\left[\Delta_{s, t} x_{n}\left(s^{\prime}, t^{\prime}\right) \mid \mathscr{G}_{s, t, \delta}^{n}\right]\right]\right| \\
& \leqslant\left(\mathrm{E}\left[\varphi^{2}\left(x_{n}\left(s_{1}, t_{1}\right), \ldots, x_{n}\left(s_{m}, t_{m}\right)\right)\right]^{1 / 2}\left(\mathrm{E}\left[Y_{n}^{2}\right]\right)^{1 / 2} \leqslant K\left(\mathrm{E}\left[Y_{n}^{2}\right]\right)^{1 / 2},\right.
\end{aligned}
$$

where $\mathscr{G}_{s, t, \delta}^{n}=\mathscr{F}_{S, t-\delta}^{n} \vee \mathscr{F}_{s-\delta, T}^{n}$ and

$$
Y_{n}:=\mathrm{E}\left[n \int_{\left[s, s^{\prime}\right] \times\left[t, t^{\prime}\right]} \sqrt{x y}(-1)^{N_{n}(x, y)} \mathrm{d} x \mathrm{~d} y \mid \mathscr{G}_{s, t, \delta}^{n}\right] .
$$

Thus, it suffices to prove that $Y_{n}$ converges to zero in $L^{2}$ when $n$ goes to infinity. Observe that $\Delta_{s-\delta, t-\delta} N_{n}(s, t)$ is independent of $\mathscr{G}_{s, t, \delta}^{n}$, and

$$
\begin{aligned}
\mathrm{E}\left[n \int_{\left[s, s^{\prime}\right] \times\left[t, t^{\prime}\right]} \sqrt{x y}(-1)^{N_{n}(x, y)} \mathrm{d} x \mathrm{~d} y \mid \mathscr{S}_{s, t, \delta}^{n}\right] \\
=\mathrm{E}\left[(-1)^{\Delta_{s-\delta, t-\delta} N_{n}(s, t)}\right] \mathrm{E}\left[n \int_{\left[s, s^{\prime}\right] \times\left[t, t^{\prime}\right]} \sqrt{x y}(-1)^{N_{n}(x, y)-\Delta_{s-\delta, t-\delta} N_{n}(s, t)} \mathrm{d} x \mathrm{~d} y \mid \mathscr{G}_{s, t, \delta}^{n}\right],
\end{aligned}
$$

which clearly goes to zero in $L^{2}$ because the conditional expectation is $L^{2}$-bounded by Lemma 3.2, and $\mathrm{E}\left[(-1)^{\Delta_{s-\delta, t-\delta} N_{n}(s, t)}\right]=\exp \left[-2 \delta^{2} n\right]$ which tends to zero as $n \rightarrow \infty$.

Proof of Proposition 4.3. We have to prove that for all $\left(s_{1}, t_{1}\right), \ldots,\left(s_{m}, t_{m}\right)$ with $s_{i} \leqslant S$, $t_{i} \leqslant t$ or $s_{i} \leqslant s, t_{i} \leqslant T$, for $i=1, \ldots, m$, and for all bounded continuous $\varphi: \mathbb{R}^{m} \rightarrow \mathbb{R}$,

$$
\mathrm{E}_{P}\left[\varphi\left(X_{s_{1}, t_{1}}, \ldots, X_{s_{m}, t_{m}}\right)\left(\left(\Delta_{s, t} X_{s^{\prime}, t^{\prime}}\right)^{2}-\left(s^{\prime}-s\right)\left(t^{\prime}-t\right)\right)\right]=0 .
$$

Recall that it suffices to prove it for $s, t>0$.

Since $P_{n}$ converges weakly to $P$ and using Lemma 3.1 it is enough to check that

$$
\mathrm{E}\left[\varphi\left(x_{n}\left(s_{1}, t_{1}\right), \ldots, x_{n}\left(s_{m}, t_{m}\right)\right)\left(\left(\Delta_{s, t} x_{n}\left(s^{\prime}, t^{\prime}\right)\right)^{2}-\left(s^{\prime}-s\right)\left(t^{\prime}-t\right)\right)\right]
$$

converges to zero when $n$ tends to infinity. But this last expression is equal to

$$
\mathrm{E}\left[\varphi\left(x_{n}\left(s_{1}, t_{1}\right), \ldots, x_{n}\left(s_{m}, t_{m}\right)\right)\left(\mathrm{E}\left[\left(\Delta_{s, t} x_{n}\left(s^{\prime}, t^{\prime}\right)\right)^{2} \mid \mathscr{F}_{S, t}^{n} \vee \mathscr{F}_{s, T}^{n}\right]-\left(s^{\prime}-s\right)\left(t^{\prime}-t\right)\right)\right] .
$$

Finally, in order to prove that this expression tends to zero, it suffices to show that,

$$
\mathrm{E}\left[\left(\Delta_{s, t} x_{n}\left(s^{\prime}, t^{\prime}\right)\right)^{2} \mid \mathscr{F}_{S, t}^{n} \vee \mathscr{F}_{s, T}^{n}\right] \stackrel{L^{2}}{\rightarrow}\left(s^{\prime}-s\right)\left(t^{\prime}-t\right) \quad \text { as } n \rightarrow \infty .
$$

This last convergence can be done using the following two facts.

Fact 1. $\mathrm{E}\left[\mathrm{E}\left[\left(\Delta_{s, t} x_{n}\left(s^{\prime}, t^{\prime}\right)\right)^{2} \mid \mathscr{F}_{S, t}^{n} \vee \mathscr{F}_{s, T}^{n}\right]\right]=\mathrm{E}\left[\left(\Delta_{s, t} x_{n}\left(s^{\prime}, t^{\prime}\right)\right)^{2}\right] \rightarrow\left(s^{\prime}-s\right)\left(t^{\prime}-t\right)$, as $n \rightarrow$ $\infty$.

This result is proved in Lemma 4.4. 
Fact 2. There exist some constants $C_{n}$ converging to $\left(s^{\prime}-\right)^{2}\left(t^{\prime}-t\right)^{2}$, when $n$ goes to infinity, such that

$$
\mathrm{E}\left[\mathrm{E}\left[\left(\Delta_{s, t} x_{n}\left(s^{\prime}, t^{\prime}\right)\right)^{2} \mid \mathscr{F}_{S, t}^{n} \vee \mathscr{F}_{s, T}^{n}\right]\right]^{2} \leqslant C_{n}
$$

This result is proved in Lemma 4.5.

Facts 1 and 2 imply the convergence stated in (2) because if we assume that they are true,

$$
\begin{aligned}
0 & \leqslant \mathrm{E}\left[\mathrm{E}\left[\left(\Delta_{s, t} x_{n}\left(s^{\prime}, t^{\prime}\right)\right)^{2} \mid \mathscr{F}_{S, t}^{n} \vee \mathscr{F}_{s, T}^{n}\right]-\left(s^{\prime}-s\right)\left(t^{\prime}-t\right)\right]^{2} \\
& \leqslant C_{n}-2\left(s^{\prime}-s\right)\left(t^{\prime}-t\right) \mathrm{E}\left[\left(\Delta_{s, t} x_{n}\left(s^{\prime}, t^{\prime}\right)\right)^{2}\right]+\left(s^{\prime}-s\right)^{2}\left(t^{\prime}-t\right)^{2},
\end{aligned}
$$

and the right-hand side of this expression obviously converges to 0 . This finishes the proof of Proposition 4.3.

Lemma 4.4. In the previous situation

$$
\lim _{n \rightarrow \infty} \mathrm{E}\left[\left(\Delta_{s, t} x_{n}\left(s^{\prime}, t^{\prime}\right)\right)^{2}\right]=\left(s^{\prime}-s\right)\left(t^{\prime}-t\right) .
$$

Proof. In the proof of Lemma 3.2 we have shown that

$$
\mathrm{E}\left[\left(\Delta_{s, t} x_{n}\left(s^{\prime}, t^{\prime}\right)\right)^{2}\right]=2\left(I_{1}+I_{2}\right),
$$

where

$$
\begin{aligned}
I_{1}= & n^{2} \int_{\left[s, s^{\prime}\right]^{2} \times\left[t, t^{\prime}\right]^{2}} \sqrt{x_{1} x_{2} y_{1} y_{2}} \exp \left[-2 n\left(x_{2} y_{2}-x_{1} y_{1}\right)\right] I_{\left\{x_{1} \leqslant x_{2}\right\}} I_{\left\{y_{1} \leqslant y_{2}\right\}} \mathrm{d} x_{1} \mathrm{~d} x_{2} \mathrm{~d} y_{1} \mathrm{~d} y_{2}, \\
I_{2}= & n^{2} \int_{\left[s, s^{\prime}\right]^{2} \times\left[t, t^{\prime}\right]^{2}} \sqrt{x_{1} x_{2} y_{1} y_{2}} \exp \left[-2 n\left(x_{2}-x_{1}\right) y_{1}-2 n\left(y_{2}-y_{1}\right) x_{1}\right] \\
& \times I_{\left\{x_{1} \leqslant x_{2}\right\}} I_{\left\{y_{1} \leqslant y_{2}\right\}} \mathrm{d} x_{1} \mathrm{~d} x_{2} \mathrm{~d} y_{1} \mathrm{~d} y_{2} .
\end{aligned}
$$

We can write the integral $I_{2}$ as

$$
\begin{aligned}
I_{2}= & \int_{s}^{s^{\prime}} \frac{1}{2 \sqrt{x_{1}}} \int_{t}^{t^{\prime}} \frac{1}{2 \sqrt{y_{1}}}\left[\int_{y_{1}}^{t^{\prime}}\left(2 n x_{1} \exp \left[-2 n x_{1}\left(y_{2}-y_{1}\right)\right]\right) \sqrt{y_{2}} \mathrm{~d} y_{2}\right. \\
& \left.\times \int_{x_{1}}^{s^{\prime}}\left(2 n y_{1} \exp \left[-2 n y_{1}\left(x_{2}-x_{1}\right)\right]\right) \sqrt{x_{2}} \mathrm{~d} x_{2}\right] \mathrm{d} y_{1} \mathrm{~d} x_{1} .
\end{aligned}
$$

The last integral tends to $\sqrt{x_{1}}$ because $2 n y_{1} \exp \left[-2 n y_{1}\left(x_{2}-x_{1}\right)\right]$ is a probability density that gives an approximation of the identity as $n \rightarrow \infty$, and the penultimate tends to $\sqrt{y_{1}}$ as $n \rightarrow \infty$. The convergence is bounded, because the two integrals are bounded by $\sqrt{s^{\prime}}$ and $\sqrt{t^{\prime}}$ respectively and $1 / \sqrt{x_{1} y_{1}}$ is integrable, so, by the dominated convergence theorem, we obtain 


$$
\lim _{n \rightarrow \infty} I_{2}=\frac{1}{4} \int_{s}^{s^{\prime}} \int_{t}^{t^{\prime}} \mathrm{d} y_{1} \mathrm{~d} x_{1}=\frac{\left(s^{\prime}-s\right)\left(t^{\prime}-t\right)}{4} .
$$

In the same way,

$$
\begin{aligned}
I_{1}= & \int_{s}^{s^{\prime}} \frac{1}{2 \sqrt{x_{2}}} \int_{t}^{t^{\prime}} \frac{1}{2 \sqrt{y_{1}}}\left[\int_{y_{1}}^{t^{\prime}}\left(2 n x_{2} \exp \left[-2 n x_{2}\left(y_{2}-y_{1}\right)\right]\right) \sqrt{y_{2}} \mathrm{~d} y_{2}\right. \\
& \left.\times \int_{s}^{x_{2}}\left(2 n y_{1} \exp \left[-2 n y_{1}\left(x_{2}-x_{1}\right)\right]\right) \sqrt{x_{1}} \mathrm{~d} x_{1}\right] \mathrm{d} y_{1} \mathrm{~d} x_{2},
\end{aligned}
$$

and we have

$$
\lim _{n \rightarrow \infty} I_{1}=\frac{\left(s^{\prime}-s\right)\left(t^{\prime}-t\right)}{4}
$$

Lemma 4.5. In the previous situation there exist some constants $C_{n}$ converging to $\left(s^{\prime}-s\right)^{2}\left(t^{\prime}-t\right)^{2}$ when $n$ tends to infinity, such that

$$
\mathrm{E}\left[\mathrm{E}\left(\left(\Delta_{s, t} x_{n}\left(s^{\prime}, t^{\prime}\right)\right)^{2} \mid \mathscr{F}_{S, t}^{n} \vee \mathscr{F}_{s, T}^{n}\right)\right]^{2} \leqslant C_{n} .
$$

Proof. We recall that it suffices to prove the case $s, t>0$. Thus, we will suppose from now on that $s, t>0$. By measurability and independence, we have that

$$
\begin{aligned}
& \mathrm{E}\left(\left(\Delta_{s, t} x_{n}\left(s^{\prime}, t^{\prime}\right)\right)^{2} \mid \mathscr{F}_{S, t}^{n} \vee \mathscr{F}_{s, T}^{n}\right) \\
& \quad=n^{2} \int_{\left[s, s^{\prime}\right]^{2} \times\left[t, t^{\prime}\right]^{2}}(-1)^{\sum_{i=1}^{2}\left(\Delta_{0, t} N_{n}\left(s, y_{i}\right)+\Delta_{s, 0} N_{n}\left(x_{i}, t\right)\right)} \times \prod_{i=1}^{2} \sqrt{x_{i} y_{i}} \mathrm{E}\left[(-1)^{\sum_{i=1}^{2} \Delta_{s, t} N_{n}\left(x_{i}, y_{i}\right)}\right] \mathrm{d} x_{1} \ldots \mathrm{d} y_{2} ;
\end{aligned}
$$

then

$$
\begin{aligned}
\mathrm{E}\left[\mathrm{E}\left(\left(\Delta_{s, t} x_{n}\left(s^{\prime}, t^{\prime}\right)\right)^{2} \mid \mathscr{F}_{S, t}^{n} \vee \mathscr{F}_{s, T}^{n}\right)\right]^{2} \\
=\mathrm{E}\left[n^{4} \int_{\left[s, s^{\prime}\right]^{4} \times\left[t, t^{\prime}\right]^{4}}(-1)^{\sum_{i=1}^{4}\left(\Delta_{0, t} N_{n}\left(s, y_{i}\right)+\Delta_{s, 0} N_{n}\left(x_{i}, t\right)\right)}\right. \\
\left.\quad \times \prod_{i=1}^{4} \sqrt{x_{i} y_{i}} \mathrm{E}\left[(-1)^{\sum_{i=1}^{2} \Delta_{s, t} N_{n}\left(x_{i}, y_{i}\right)}\right] \mathrm{E}\left[(-1)^{\sum_{i=3}^{4} \Delta_{s, t} N_{n}\left(x_{i}, y_{i}\right)}\right] \mathrm{d} x_{1} \ldots \mathrm{d} y_{4}\right] .
\end{aligned}
$$

By the arguments of the proof of Lemma 3.2, we have that

$$
\mathrm{E}\left[(-1)^{\sum_{i=1}^{2} \Delta_{s, t} N_{n}\left(x_{i}, y_{i}\right)}\right] \leqslant \exp \left[-2 n\left(\left|x_{2}-x_{1}\right|\left(\min \left\{y_{1}, y_{2}\right\}-t\right)+\left|y_{2}-y_{1}\right|\left(\min \left\{x_{2}, x_{2}\right\}-s\right)\right)\right],
$$

and that 


$$
\mathrm{E}\left[(-1)^{\sum_{i=3}^{4} \Delta_{s, t} N_{n}\left(x_{i}, y_{i}\right)}\right] \leqslant \exp \left[-2 n\left(\left|x_{4}-x_{3}\right|\left(\min \left\{y_{3}, y_{4}\right\}-t\right)+\left|y_{4}-y_{3}\right|\left(\min \left\{x_{3}, x_{4}\right\}-s\right)\right)\right]
$$

Then,

$$
\begin{aligned}
& \mathrm{E}\left[\mathrm{E}\left(\left(\Delta_{s, t} x_{n}\left(s^{\prime}, t^{\prime}\right)\right)^{2} \mid \mathscr{F}_{S, t}^{n} \vee \mathscr{F}_{s, T}^{n}\right)\right]^{2} \\
& \leqslant 16 n^{4} \int_{\left[s, s^{\prime}\right]^{4} \times\left[t, t^{\prime}\right]^{4}}\left|\mathrm{E}(-1)^{\sum_{i=1}^{4}\left(\Delta_{0, t} N_{n}\left(s, y_{i}\right)+\Delta_{s, 0} N_{n}\left(x_{i}, t\right)\right)}\right| \\
& \times \prod_{i=1}^{4} \sqrt{x_{i} y_{i}} \exp \left[-2 n\left(\left(x_{2}-x_{1}\right)\left(y_{1}-t\right)\left(y_{2}-y_{1}\right)\left(x_{1}-s\right)\right)\right] \\
& \times \exp \left[-2 n\left(\left(x_{4}-x_{3}\right)\left(y_{3}-t\right)+\left(y_{4}-y_{3}\right)\left(x_{3}-s\right)\right)\right] \\
& \times I_{\left\{x_{1} \leqslant x_{2}\right\}} I_{\left\{y_{1} \leqslant y_{2}\right\}} I_{\left\{x_{3} \leqslant x_{4}\right\}} I_{\left\{y_{3} \leqslant y_{4}\right\}} \mathrm{d} x_{1} \ldots \mathrm{d} y_{4} .
\end{aligned}
$$

We can divide the last integral in two parts: the integral over $A=\left(\left\{y_{1} \leqslant y_{2} \leqslant y_{3} \leqslant y_{4}\right\}\right.$ $\left.\cup\left\{y_{3} \leqslant y_{4} \leqslant y_{1} \leqslant y_{2}\right\}\right) \cap\left(\left\{x_{1} \leqslant x_{2} \leqslant x_{3} \leqslant x_{4}\right\} \cup\left\{x_{3} \leqslant x_{4} \leqslant x_{1} \leqslant x_{2}\right\}\right)$; and the integral over $A^{c}$.

If we integrate over $A$,

$$
\begin{aligned}
&\left|\mathrm{E}(-1)^{\sum_{i=1}^{4}\left(\Delta_{0, t} N_{n}\left(s, y_{i}\right)+\Delta_{s, 0} N_{n}\left(x_{i}, t\right)\right)}\right| \\
& \quad=\exp \left[-2 n s\left[\left(y_{4}-y_{3}\right)+\left(y_{2}-y_{1}\right)\right]-2 n t\left[\left(x_{4}-x_{3}\right)+\left(x_{2}-x_{1}\right)\right]\right],
\end{aligned}
$$

and the integral given in (5), over $A$, can be bounded by

$16\left(n^{2} \int_{\left[s, s^{\prime}\right]^{2} \times\left[t, t^{\prime}\right]^{2}} \prod_{i=1}^{2} \sqrt{x_{i} y_{i}} \exp \left[-2 n\left(x_{2}-x_{1}\right) y_{1}-2 n\left(y_{2}-y_{1}\right) x_{1}\right] I_{\left\{x_{1} \leqslant x_{2}\right\}} I_{\left\{y_{1} \leqslant y_{2}\right\}} \mathrm{d} x_{1} \ldots \mathrm{d} y_{2}\right)^{2}$

which, as we have shown in Lemma 4.4, converges to

$$
16\left(\frac{\left(s^{\prime}-s\right)\left(t^{\prime}-t\right)}{4}\right)^{2}=\left(s^{\prime}-s\right)^{2}\left(t^{\prime}-t\right)^{2}
$$

When we integrate over $A^{c}$, the integral converges to zero. Indeed, if we have $y_{1} \leqslant y_{3} \leqslant y_{2} \leqslant y_{4}$ (or $y_{1} \leqslant y_{3} \leqslant y_{4} \leqslant y_{2}$ ) we can bound (3) by $\exp \left[-2 n\left(y_{2}-y_{3}\right)\left(x_{1}-s\right)\right]$ (or $\exp \left[-2 n\left(y_{4}-y_{3}\right)\left(x_{1}-s\right)\right]$ ) and (4) by 1 .

If we have $y_{3} \leqslant y_{1} \leqslant y_{4} \leqslant y_{2}$ (or $y_{3} \leqslant y_{1} \leqslant y_{2} \leqslant y_{4}$ ) we likewise bound (4) by $\exp \left[-2 n\left(y_{4}-y_{1}\right)\left(x_{3}-s\right)\right]\left(\right.$ or $\left.\exp \left[-2 n\left(y_{2}-y_{1}\right)\left(x_{3}-s\right)\right]\right)$ and (3) by 1 .

Then, by doing a change of variables in order to obtain $x_{1} \leqslant x_{2} \leqslant x_{3} \leqslant x_{4}$ and $y_{1} \leqslant y_{2} \leqslant y_{3} \leqslant y_{4}$, we majorize the integral given in (5), over $\left(\left\{y_{1} \leqslant y_{2} \leqslant y_{3} \leqslant y_{4}\right\} \cup\right.$ $\left.\left\{y_{3} \leqslant y_{4} \leqslant y_{1} \leqslant y_{2}\right\}\right)^{c}$, by 


$$
\begin{aligned}
& K n^{4} \int_{\left[s, s^{\prime}\right]^{4} \times\left[t, t^{\prime}\right]^{4}} \prod_{i=1}^{4} \sqrt{x_{i} y_{i}} \exp \left[-2 n t\left[\left(x_{4}-x_{3}\right)+\left(x_{2}-x_{1}\right)\right]-2 n s\left[\left(y_{4}-y_{3}\right)+\left(y_{2}-y_{1}\right)\right]\right. \\
& \left.\quad-2 n\left(y_{3}-y_{2}\right)\left(x_{1}-s\right)\right] I_{\left\{x_{1} \leqslant x_{2} \leqslant x_{3} \leqslant x_{4}\right\}} I_{\left\{y_{1} \leqslant y_{2} \leqslant y_{3} \leqslant y_{4}\right\}} \mathrm{d} x_{1} \ldots \mathrm{d} y_{4} .
\end{aligned}
$$

Finally, using the fact that $s, t>0$, this integral equals to

$$
\begin{aligned}
& K \frac{1}{s^{2} t^{2}} \int_{s}^{s^{\prime}} \sqrt{x_{1}} \int_{s}^{s^{\prime}} \sqrt{x_{3}} \int_{t}^{t^{\prime}} \sqrt{y_{2}} \int_{y_{2}}^{t^{\prime}} \sqrt{y_{3}}\left\{\int_{x_{3}}^{s^{\prime}} 2 n t \exp \left[-2 n t\left(x_{4}-x_{3}\right)\right] \sqrt{x_{4}} \mathrm{~d} x_{4}\right. \\
& \quad \times \int_{x_{1}}^{s^{\prime}} 2 n t \exp \left[-2 n t\left(x_{2}-x_{1}\right)\right] \sqrt{x_{2}} \mathrm{~d} x_{2} \int_{y_{3}}^{t^{\prime}} 2 n s \exp \left[-2 n s\left(y_{4}-y_{3}\right)\right] \sqrt{y_{4}} \mathrm{~d} y_{4} \\
& \left.\quad \times \int_{t}^{y_{2}} 2 n s \exp \left[-2 n s\left(y_{2}-y_{1}\right)\right] \sqrt{y_{1}} \mathrm{~d} y_{1}\right\} \exp \left[-2 n\left(y_{3}-y_{2}\right)\left(x_{1}-s\right)\right] \mathrm{d} y_{3} \mathrm{~d} y_{2} \mathrm{~d} x_{3} \mathrm{~d} x_{1},
\end{aligned}
$$

which goes to zero by dominated convergence.

Interchanging the roles of the variables $x_{i}$ and the $y_{i}$ for $i=1, \ldots, 4$, we will obtain a similar integral.

This completes the proof.

\section{Acknowledgements}

The authors are greatly indebted to a referee for several helpful suggestions. This work was partially supported by grants CICYT PB96-1182, CICYT PB96-0088 and CIRIT 1997SGR00144.

\section{References}

Bickel, P.J. and Wichura, M.J. (1971) Convergence criteria for multiparameter stochastic processes and some applications. Ann. Math. Statist., 42, 1656-1670.

Cairoli, R. and Walsh, J.B. (1975) Stochastic integrals in the plane. Acta Math., 134, 111-183.

Florit, C. and Nualart, D. (1996) Diffusion approximation for hyperbolic stochastic differential equations. Stochastic Process. Appl., 65, 1-15.

Stroock, D. (1982) Lectures on Topics in Stochastic Differential Equations. Berlin: Springer-Verlag. Tudor, C. (1980) Remarks on the martingale problem in the two dimensional time parameter. Rev. Roumaine Math. Pures Appl., 25, 1551-1556.

Received January 1998 and revised March 1999 